\title{
O PROBLEMA DO CARPINTEIRO: ESTUDANDO SEMELHANÇA DE TRIÂNGULOS POR MEIO DA FACHADA DE UMA CASA
}

\author{
Márcio Antônio Souza Paim
}

\begin{abstract}
RESUMO
Este trabalho tem o objetivo de investigar o uso das Tecnologias de Informação e Comunicação (TICs) como recursos didáticos no processo de aprendizagem da matemática na escola básica. Foi realizada uma sequência de ensino utilizando o software Régua e Compasso (R.e.C.) para a compreensão da semelhança de triângulos em uma turma da Educação de Jovens e Adultos - EJA. O $1^{\circ}$ módulo do curso em Higiene e Segurança do Trabalho foi em uma Instituição Técnica Federal do município de Santo Amaro, Estado da Bahia. A sequência foi construída por meio de uma atividade que explora uma situação cotidiana sobre o conteúdo proposto a partir da ideia de construção do conhecimento do aluno, através das Tecnologias de Informação e de tarefas implementadas pelo professor. A análise final sugere que, ao investigar os registros de uma aluna, de uma turma de quinze estudantes, na resolução da atividade com o auxílio do software R.e.C., a mesma desenvolveu uma melhor aprendizagem dos conceitos sobre semelhança de triângulos. Este trabalho mostrou que a descoberta e o uso dessas tecnologias podem ser úteis na contextualização do ensino de matemática, contribuindo para a aprendizagem dos estudantes.
\end{abstract}

Palavras-chave: Semelhança de Triângulos. EJA. R.e.C.

\begin{abstract}
This work aims to investigate the use of Information and Communication Technologies (ICTs) as teaching resources in mathematics learning process, in basic school. A teaching sequence using the Ruler and Compass software (C.a.R.), for understanding the similarity of triangles in a class of Youth and Adult Education - YAE was held. The $1^{\text {st }}$ course module on Hygiene and Safety was in a Federal Technical Institution in Santo Amaro, Bahia. The sequence was constructed through an activity that explores an everyday situation on the proposed content, from the construction idea of student's knowledge through information technologies and tasks implemented by the teacher. The final analysis suggests that, when investigating the records of one student, in a class of fifteen, in solving activity with the help of C.a.R. software, she developed a better learning of the concepts of similar triangles. This work showed that the discovery and the use of these technologies may be useful in contextualizing the teaching of mathematics, contributing to student learning.
\end{abstract}

Keywords: Similarity of Triangles. YAE. C.a.R.

\footnotetext{
${ }^{1}$ Especialização em Novas tecnologias para o ensino da matemática pela Universidade Federal Fluminense e atualmente é professor do Instituto Federal da Bahia (IFBA) - Campus de Santo Amaro. E-mail: maspaim@hotmail.com.
} 


\section{INTRODUÇÃO}

Com o crescimento dos programas sociais e a necessidade de um ensino mais contextualizado, centrado em grupos humanos, a possibilidade de resolução de alguns problemas da vida diária, através de conceitos matemáticos, trouxe para o estudante da educação básica novas alternativas de aprendizagem e uma busca pelo real significado da disciplina em suas vidas.

Diversas transformações causadas pelo avanço tecnológico têm chegado ao ambiente escolar e exigido uma proposta de mudança no ensino da matemática tanto no foco da aprendizagem dos estudantes, quanto na melhor maneira que o professor tem em aproveitar as ferramentas tecnológicas disponibilizadas pelas Tecnologias de Informação.

Surgindo como uma opção para o aperfeiçoamento das técnicas de um ensino conteudista que não provoca uma reflexão sobre prática docente ou não estimula ao aluno a busca pelo seu conhecimento, as TICs têm sido um tema largamente discutido por pesquisadores do ensino da matemática. Para Valente (1999), o não uso das tecnologias, como recursos para a ampliação e criação de ambientes de aprendizado que proporcionem a construção do conhecimento, é um obstáculo para o desenvolvimento de mudanças satisfatórias nos sistemas de ensino.

Borba (2002) reflete sobre a construção do conhecimento a partir do coletivo seres humanos-com-mídias na realização de atividades em ambientes tecnológicos como o da internet. Neste caso, o saber não é produzido individualmente por seres humanos, mas, principalmente, através da interação com as novas linguagens que emergem dos computadores. Um tipo de recursos tecnológicos utilizados para fins de aprendizagem educativa é o OA.

Wiley (2000 apud BAIRRAL, 2010) define um Objeto de Aprendizagem (AO) como uma tecnologia específica que apresenta a interatividade como uma de suas características essenciais. Dentre outras particularidades, afirma ser uma ferramenta pedagógica reutilizável, ou seja, que pode ser aproveitável em outras tarefas e em outros ambientes. Corresponde a um recurso interativo que, quando adequadamente produzido, contribui para o processo de ensino e aprendizagem.

Dentro do aspecto tecnológico, o aparecimento dessas mídias possibilitou o conhecimento e prática de diversas ferramentas tecnológicas, tanto através de animações geradas por softwares dinâmicos, quanto na exploração de ferramentas dos materiais interativos criados estrategicamente para públicos específicos como a EJA.

ForSci.: r. cient. IFMG campus Formiga, Formiga, v. 3, n. 1, p. 104-121, jan./jun. 2015 
Desejando aproximar essa classe de estudantes o mais possível da sua realidade e se utilizando do software livre R.e.C., foi criado pelo professor de matemática da turma um OA que simula as dimensões de uma janela contida na fachada de uma casa em construção, para estimulá-los na resolução de atividades sobre semelhança de triângulos.

Nesse trabalho convém investigar o registro de uma estudante da EJA, de uma turma de 15 alunos, ao realizar uma atividade de matemática sobre o conteúdo semelhança de triângulos, construída com um software computacional na forma de um OA. A sequência de ensino, elaborada pelo professor de matemática da turma, foi realizada por estudantes do $1^{\circ}$ módulo do curso em Higiene e Segurança do Trabalho no Instituto Federal da Bahia, campus de Santo Amaro.

A realização da tarefa se configurou em torno das relações entre o uso das TICs e as práticas do cotidiano que podem ser levadas até a sala de aula. Entende-se que subsídios tecnológicos feitos para o ensino de matemática sejam úteis para aproximar o aluno da sua realidade, dando significado à aprendizagem.

\section{O R.e.C.}

O Régua e Compasso, abreviado por R.e.C em português e em inglês em C.a.R, foi criado e desenvolvido pelo professor René Grothmann da Universidade Católica de Berlim, na Alemanha em 1988 (ARAÚJO, 2003). De acordo com o site http://www.gregosetroianos.mat.br/softcar.asp o professor levou alguns anos, depois da popularização da linguagem Java, para desenvolvê-lo e distingui-lo das versões anteriores.

É um software de geometria dinâmica e permite que qualquer objeto plano criado dentro do seu ambiente rotacione ou se desloque de um ponto a outro. Pode ser conseguido diretamente pela internet acessando o link: http://www.professores.uff.br/hjbortol/car/ conforme Bartolossi (2010). Ao se abrir o programa, é fácil perceber a quantidade de ferramentas disponíveis, conforme FIG. 1

Dentre as quais, foram utilizadas as seguintes:

$\circ \quad=$ Ponto. $\quad \sigma^{\circ}=$ Segmento definido por dois pontos. $\quad \delta \delta=$ Ângulo com amplitude fixa. 


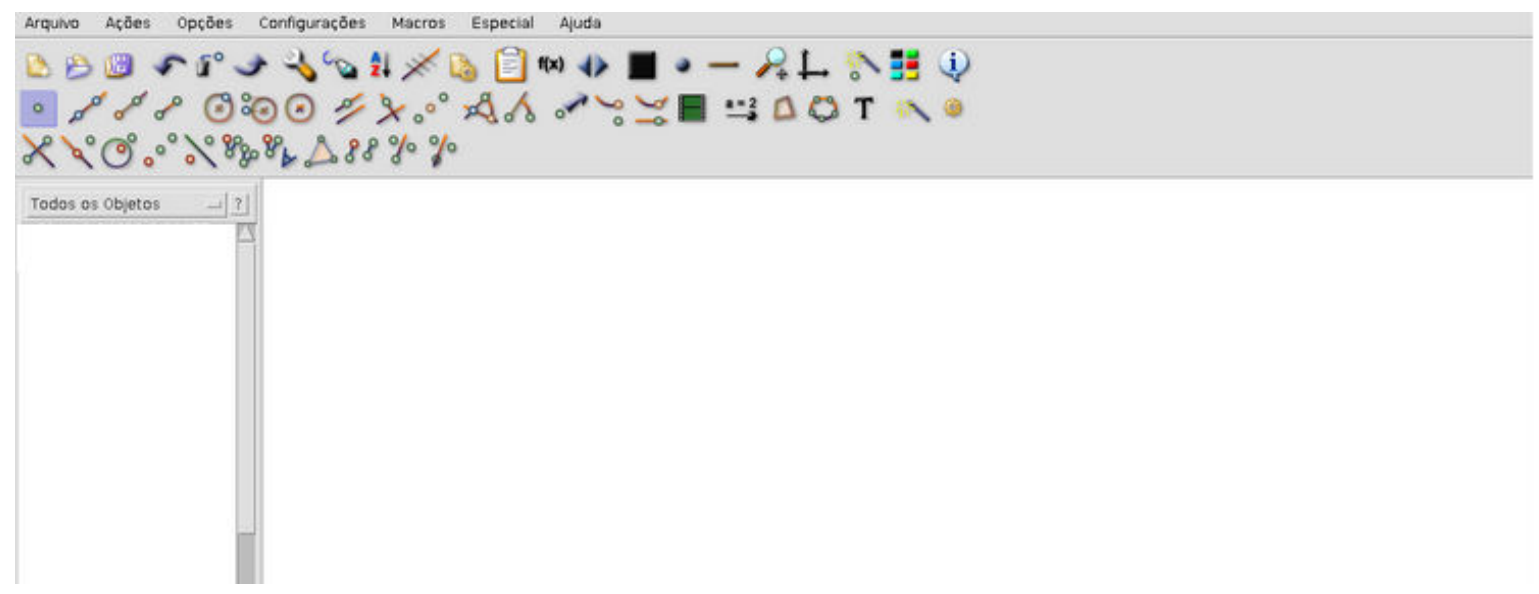

Figura 1 - Tela do R.e.C.

Fonte: Autor.

\section{CONSTRUINDO A ATIVIDADE}

Se apropriando de conceitos sobre a semelhança de triângulos, a atividade teve o propósito de obter razões de semelhança entre dois lados de triângulos semelhantes utilizando os comandos disponibilizados pelo software. Foi explorado o caso L.A.L. (lado, ângulo, lado) de semelhança de triângulos, no qual, se dois ou mais triângulos possuírem ângulos ordenadamente iguais formados entre dois lados proporcionais, então serão semelhantes.

A construção da tarefa foi baseada na observação da lateral de uma casa em construção. O seu desenho criado com o auxílio do software R.e.C. é similar ao da própria lateral da casa:

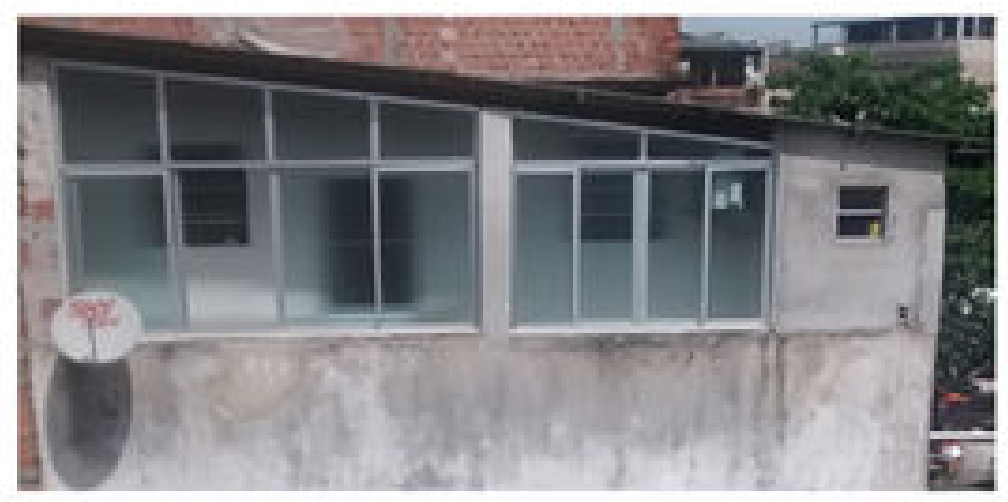

Figura 2a - Foto da lateral real da casa Fonte: Autor. 


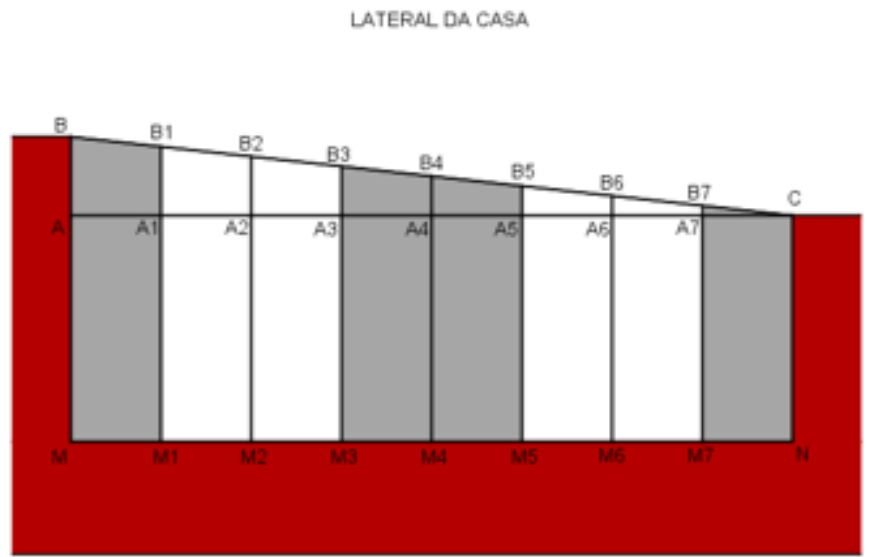

Figura $2 b-$ Desenho da lateral da casa feito com o R.e.C. Fonte: Autor.

Nas janelas que compõem a fachada da casa, podemos observar diversos segmentos de reta: $\mathrm{AM}, \mathrm{A} 1 \mathrm{M} 1, \ldots, \mathrm{AA} 1, \mathrm{MM} 1, \ldots, \mathrm{AC}, \mathrm{MN}, \ldots$, paralelos e que formam os polígonos AA1M1M, ..., ACNM, ..., entre os quais se situam triângulos ACB, A1CB1, ..., semelhantes entre si.

O software possibilita que, ao clicar com o botão direito do mouse em qualquer segmento de reta, sejam vistos os valores das medidas de cada comprimento. Sendo assim, além do ambiente computacional, foi indicado que os alunos registrassem os comprimentos obtidos no ambiente papel e lápis para fins de cálculo das razões entre dois segmentos.

A turma possui 15 alunos do curso noturno em Higiene e Segurança no Trabalho na modalidade PROEJA, também conhecida como Educação de Jovens e Adultos. Primeiramente, foi criado um questionário para analisar o conhecimento dos alunos sobre as tecnologias de informação, sobre outras tecnologias e, principalmente, sobre o software R.e.C. Os dados foram coletados por um questionário e as respostas registradas em uma tabela.

Relacionamos o conhecimento do conteúdo matemático com o uso do R.e.C. como uma tecnologia de informação (apêndice A), através das quais fez-se um levantamento inicial sobre o conhecimento e utilização da tecnologia pelos alunos dentro e fora da sala de aula. Em seguida, foram realizados na seguinte ordem um(a):

I) Pré-teste: Um exercício sobre o conteúdo matemático (semelhança de triângulos) para avaliar o conhecimento dos alunos. Esse mini teste (apêndice B) foi realizado por entender que os alunos possuíam algum conhecimento prévio sobre o assunto antes de entrar na instituição;

II) Intervenção: Aplicação da sequência de ensino com a atividade proposta e o uso do software R.e.C. 


\section{RESULTADOS E DISCUSSÕES}

Composta por 15 alunos (3 homens e 12 mulheres), a turma não apresentou resistência ou constrangimento em responder ao questionário. Entre as perguntas respondidas, está a do item VII): “AO LONGO DE SUA TRAJETÓRIA PROFISSIONAL, VOCÊ JÁ TEVE CONTATO COM SOFTWARES MATEMÁTICOS? ".

Mesmo com o avanço tecnológico e o grande acesso dos jovens aos computadores, foi constatado que o contato dos alunos com algum tipo de software matemático na sala de aula ainda continua escasso. Somente 7 alunos (2 homens e 5 mulheres) declararam ter tido algum contato com softwares matemáticos:

\section{USO DE SOFTWARES MATEMÁTICOS}

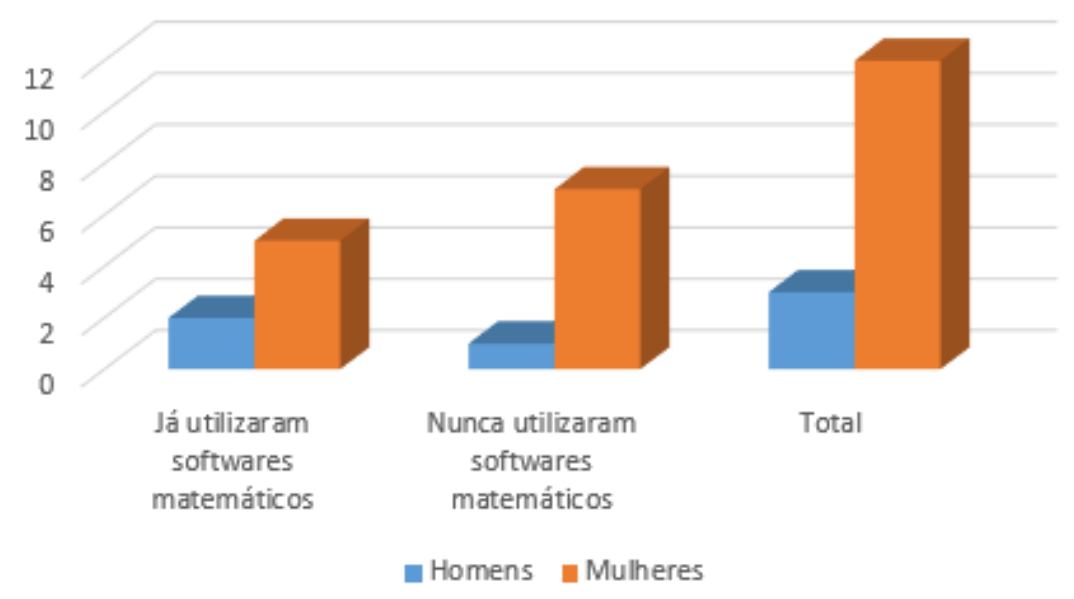

Gráfico 1 - Gráfico sobre o uso de softwares matemáticos Fonte: Autor.

Os alunos que utilizaram softwares trabalham no comércio da região, com isso tiveram contato com ferramentas tecnológicas e matemáticas, como o software excel e calculadoras científicas no trabalho e sobrevivência social, não em sala de aula. Logo, vale afirmar que todos os alunos da turma não conheciam ou nunca ouviram falar sobre o software R.e.C, sendo assim, nunca o utilizaram na sala de aula.

Entre os homens, dois trabalham por conta própria, um não trabalha. Todos apontam o uso da calculadora científica e dos computadores para projetos ou fontes de renda pessoais. Tanto os homens quanto as mulheres que exercem alguma atividade remunerada informaram a utilização do excel e das calculadoras para cálculos financeiros em seus ambientes de trabalho. 
A turma foi levada à sala de informática da instituição para uma breve explicação numa aula de 50 minutos sobre software R.e.C. Tendo em vista o desconhecimento dos alunos, o professor da disciplina realizou uma explanação sobre o que são tecnologias de informação, para que servem, quais as implicações para o ensino público e sobre as potencialidades do software em questão, orientando-os no sentido de que pudessem manipular as ferramentas contidas no software.

Depois disso, todos os alunos da turma afirmaram ter interesse em descobrir mais sobre softwares feitos para o ensino da matemática. Informaram também que a falta de conhecimento sobre as tecnologias criadas para o ensino da matemática pode estar na pouca oferta tecnológica das escolas por onde passaram antes de entrarem na instituição, alegando ser um problema de gestão de recursos das escolas adjacentes ao município que as afeta.

Portanto, não é difícil saber que, por serem da EJA, muitos desses alunos possuem necessidades e grandes dificuldades de acesso à escola. Gadotti e Romão (2011) citam que os jovens e adultos que trabalham, lutam para superar as condições de moradia, saúde, alimentação, emprego, transporte, salário e demais situações que comprometem a educação.

No pré-teste, de acordo com o enunciado, dois estudantes (2 dos 15) resolveram a proporção corretamente, observando o paralelismo entre os segmentos $\mathrm{AB}$ e $\mathrm{CD}$. Calcularam desenvolvendo a proporção 1:

$$
\frac{136}{50}=\frac{A E}{75} \Rightarrow A E=\frac{136 \times 75}{50}=204
$$

Já para os que erraram, o mesmo pré-teste mostrou erros comuns de raciocínio proporcional, como transformar uma proporção em uma equação, ex: $136+50=A E+75$.

Ao longo das aulas com o R.e.C., por meio das atividades, o simples fato do manuseio em um novo ambiente trouxe mais possibilidades de aprendizagem para os alunos dessa turma. No enunciado número 8 da atividade intitulada "o problema do carpinteiro" (ver apêndice C), ao clicar sobre o ponto de translação do "vidro" e arrastá-lo ao seu local indicado na "janela" lateral, cinco alunos (5 dos 15) constataram que as figuras planas na forma de triângulos eram semelhantes. Clicando com o botão direito do mouse sobre os segmentos que representam os lados destes triângulos, obtiveram medidas proporcionais.

$\mathrm{Na}$ figura abaixo, por exemplo, clicando sobre os segmentos $\mathrm{AC}, \mathrm{AB}, \mathrm{A} 7 \mathrm{C}$ e A7B7, tínhamos, respectivamente, o valor das seguintes medidas, mostradas na tela do software R.e.C (ver figuras 3 e 4): 


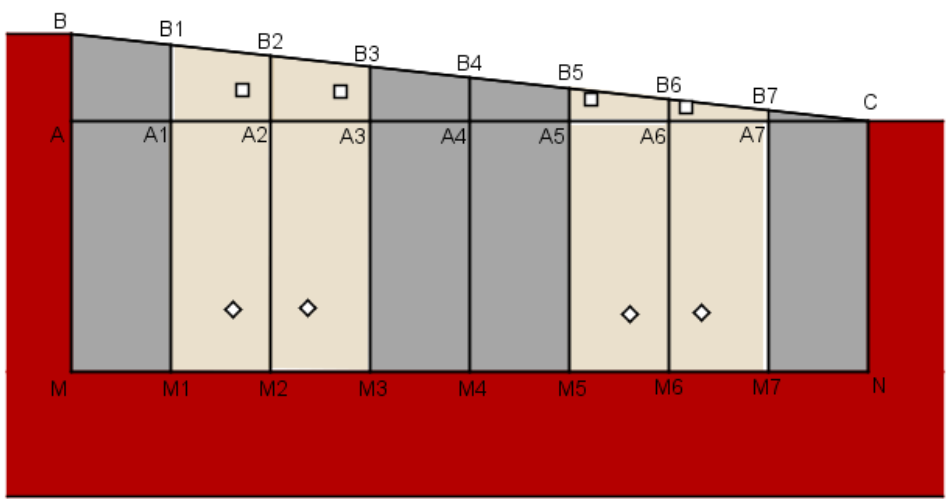

Figura 3 - O problema do carpinteiro Fonte: Autor.

\begin{tabular}{|c|c|}
\hline Comprimento & 320.0 \\
\hline Comprimento & 35.0 \\
\hline Comprimento & 40.0 \\
\hline Comprimento & 4.375 \\
\hline
\end{tabular}

Figura 4 - Medidas dos segmentos AC, AB, A7C e A7B7

Fonte: Autor.

Com as orientações do professor, $11 / 15$ dos alunos da turma observaram a similaridade dessa atividade com o pré-teste, o que facilitou a sua resolução. Desejando calcular a medida do segmento A7B7, por exemplo, utilizaram o mesmo raciocínio visto na resolução do pré-teste, ou seja, a de que, quando os lados de dois ou mais triângulos com ângulos internos ordenadamente iguais são paralelos, os seus lados serão proporcionais.

Desse modo, determinaram a medida de A7B7 da seguinte forma:

$$
\frac{320}{35}=\frac{40}{A 7 B 7} \Rightarrow A 7 B 7=\frac{35 \times 40}{320}=4,375
$$

Observa-se que esse resultado feito no ambiente "papel-lápis" confere com as informações dadas pelo software R.e.C.

Vale dizer que todos os alunos realizaram a atividade sem restrições. Isso pode indicar que manipulações geométricas com softwares de geometria dinâmica podem estimular a compreensão da matemática. Segundo Valente (1993), o auxílio das novas mídias, 
demonstrações, teoremas as aplicações dos conteúdos de Matemática, por exemplo, podem ser mais bem visualizados pelos alunos, do que se fossem só utilizados lousa e giz, como únicos materiais pedagógicos.

A seguir, destacamos o registro e escrita de algumas questões da atividade feita pela aluna A escolhida pela relevância de seus registros quando esteve em contato com o software R.e.C. Tem 18 anos de idade e soube realizar adequadamente a maioria das questões. Isso se justifica no seu entendimento sobre proporcionalidade entre as medidas dos segmentos dos triângulos semelhantes.

Nas questões números 2 e 3, conseguiu encontrar o valor dos comprimentos das medidas dos lados da "janela da lateral da casa" e completar a tabela em questão com seus cálculos proporcionais particulares, no entanto, com alguns erros: $\frac{200}{A 2 B 2}=\frac{320}{35}=21,87$, contrariando a afirmação de que $A 2 B 2=21,87$ :

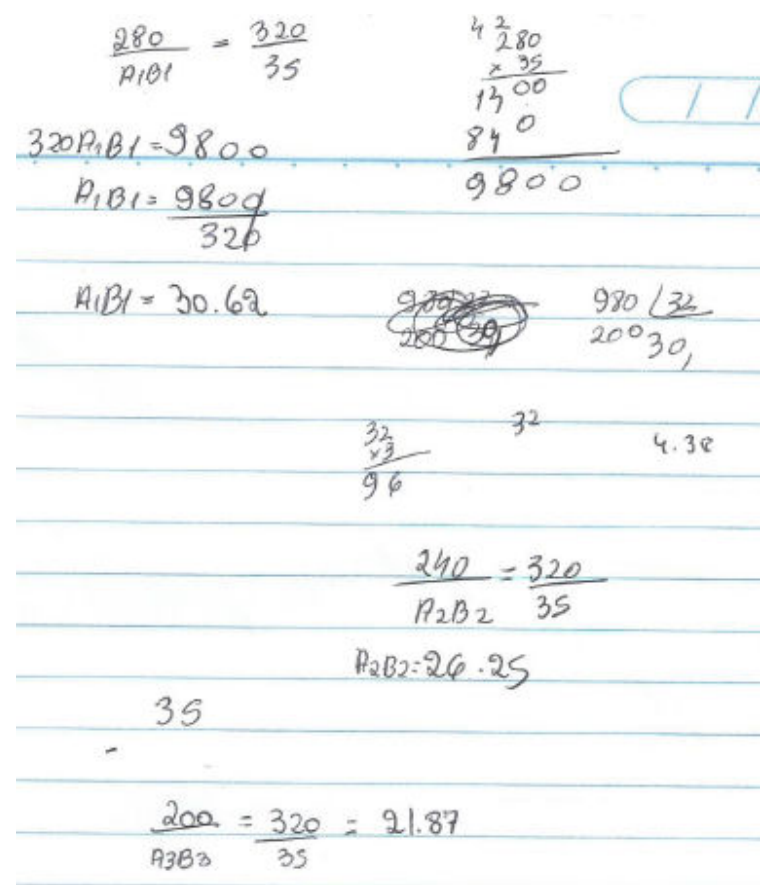

Figura 5 - Registro particular da aluna A Fonte: Dados da pesquisa.

2) Cite exemplos de segmentos de reta com a mesma medida.
$A_{1} A_{1} A_{1} A_{2}, A_{2} A_{3}, A_{3} A_{4}, A_{4} A_{5}, A 5 A_{6}, A_{6}, A_{7}, A_{7} C$

Figura 6 - Resposta correta da aluna A, questão 3 Fonte: Dados da pesquisa. 
Contrariando o enunciado escreveu as medidas com duas casas decimais:

3) Complete a tabela abaixo de acordo com alguns dos valores obtidos anteriormente, com uma casa decimal.

\begin{tabular}{|c|c|c|c|c|c|c|}
\hline segmentos & $\mathrm{AB}$ & $\mathrm{A} 1 \mathrm{~B} 1$ & $\mathrm{~A} 2 \mathrm{~B} 2$ & $\mathrm{~A} 3 \mathrm{~B} 3$ & $\mathrm{~A} 4 \mathrm{~B} 4$ & $\mathrm{~A} 5 \mathrm{~B} 5$ \\
\hline comprimentos & 35 & 30.62 & 26.25 & 21.87 & 17.49 & 13.11 \\
\hline
\end{tabular}

Figura 7 - Resposta da aluna A, questão 3).

Fonte: Dados da pesquisa.

Por outro lado, na questão número 4, não completando a tabela, apresentou dificuldades ao não perceber o ângulo BĈA comum às medidas $\mathrm{BC}, \mathrm{B} 1 \mathrm{C}, \mathrm{B} 2 \mathrm{C}, \ldots . ., \mathrm{B} 5 \mathrm{C}$ e $\mathrm{AC}, \mathrm{A} 1 \mathrm{C}, \mathrm{A} 2 \mathrm{C}, \ldots, \mathrm{A} 5 \mathrm{C}$ da "janela da lateral da casa":

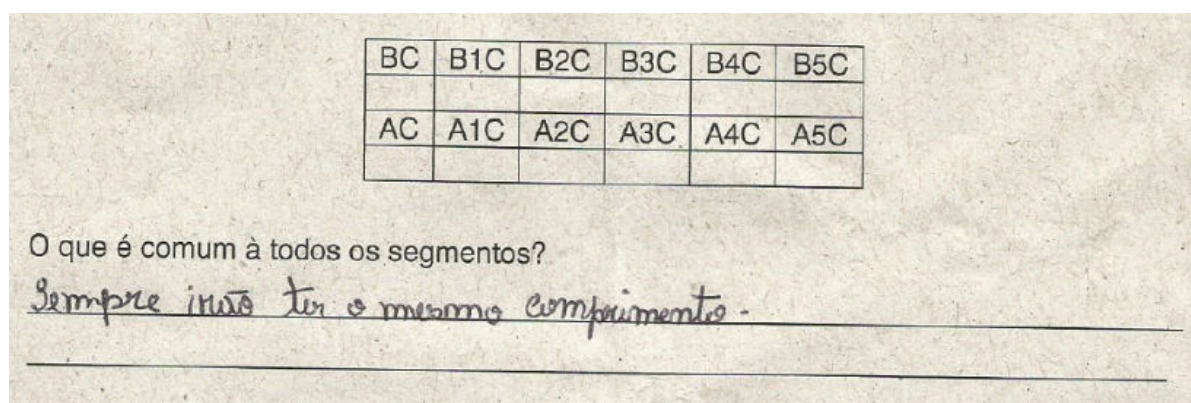

Figura 8 - Resposta da aluna A, questão 4

Fonte: Dados da pesquisa.

\section{CONCLUSÃO}

Com o auxílio do software computacional R.e.C., os alunos identificaram a proporção entre as medidas, aproximadamente iguais, dos lados de triângulos semelhantes. Explorando a atividade contextualizada sobre semelhança de triângulos, eles escreveram os seus registros no ambiente papel e lápis a partir da observação do objeto de aprendizagem no ambiente tecnológico.

As falas da aluna, do tipo: "Essa fachada da parede do desenho parece com a parede da minha casa" ou "Para achar as medidas dos lados dos triângulos, é só dividir os segmentos na mesma razão" mostram a relação do problema proposto com a sua realidade, dando-lhe uma ideia do significado da razão de semelhança entre triângulos.

Vimos a necessidade de realçar somente a aluna A pelo destaque das suas respostas, no entanto, isso não significa que os dados dos alunos restantes foram excluídos, pelo contrário, não foram descartados. Por outro lado, acreditamos que não está no alcance deste artigo tratar de maneira exaustiva a análise destes dados, os quais poderão ser abordados em outro trabalho. 
Considerando os ângulos dos triângulos ordenadamente iguais entre si, as razões representaram números reais iguais. É razoável supor que o estudante do EJA em questão, mesmo com várias dificuldades, já mencionadas, pôde verificar que a razão entre duas medidas através de um triângulo foi constante.

De uma maneira geral, foi possível perceber que as atividades criadas causaram nos estudantes uma predisposição em manusear as ferramentas em um novo ambiente de aprendizagem nunca visto antes por eles, dando-lhes condições de inventividade e construção de conhecimentos sem a ajuda do professor, como realizar proporções simples com as medidas dos lados dos triângulos, trapézios e retângulos representados pelos vidros das janelas da figura da atividade.

A importância deste trabalho está em provocar uma reflexão sobre os benefícios de práticas pedagógicas que utilizam softwares educacionais para o ensino de Matemática, buscando investigar como o uso de recursos computacionais podem favorecer a uma aprendizagem mais significativa e dinâmica na construção de conceitos matemáticos por parte dos alunos da escola básica.

\section{REFERÊNCIAS}

ARAÚJO, C. C. de. (Java) C.a.R., 2003. Disponível em: <http://www.gresetroianos. mat.br/softcar.asp>. Acesso em: 06 jun. 2015.

BAIRRAL, M. de A. A Educação Matemática em ambientes virtuais. In: ENCONTRO NACIONAL DE EDUCAÇÃO MATEMÁTICA, X., 2010, Salvador. Anais... Salvador: [s.n.], 07 - 09 jul. 2010.

BARTOLOSSI, H. J. Régua e Compasso, 2010. Disponível em:

$<$ http://www.professores.uff.br/hjbortol/car/>. Acesso em: 06 jun. 2015.

BORBA, M. de C. Coletivos seres-humanos-com-mídias e a produção de Matemática. In: SIMPÓSIO BRASILEIRO DE PSICOLOGIA DA EDUCAÇÃO MATEMÁTICA,. I, 2002, Curitiba. Anais... Curitiba: UFPR, PUCPR, Universidade Tuiuti do Paraná, 2002.

GADOTTI, M.; ROMÃO, J. Educação de jovens e adultos: teoria, prática e proposta. São Paulo: Cortez, 2011.

IEZZI, G. Fundamentos da Matemática Elementar: volume 9. São Paulo: Atual, 1993. 
VALENTE, J. A. O computador na sociedade do conhecimento. Campinas, SP: Unicamp/NIED, 1999.

Por que o computador na educação? In: VALENTE, J. A. (Org.). Computadores e

Conhecimento: repensando a educação. Campinas: Unicamp/Nied, 1993, p. 24 - 44.

Recebido em: 18/05/2015

Aprovado em: 16/06/2015

Publicado em: 29/06/2015 


\section{APÊNDICE A - Questionário Inicial}

\section{QUESTIONÁRIO}

Caro(a) Aluno(a), estamos desenvolvendo uma pesquisa, cujo objetivo geral é "Compreender a semelhança de triângulos por meio de software R.e.C". Para isso, um dos instrumentos de coleta de dados será este questionário. Assim, contando com a sua colaboração, solicitamos o seu preenchimento*.

Atenciosamente,

* O preenchimento deste questionário autoriza a divulgação de suas informações. Porém, nome e contatos não serão divulgados em nenhum momento, em respeito à sua privacidade.

\section{IDENTIFICAÇÃO}

NOME: A1

I) SEXO:

a) ( ) FEMININO

b) ( ) MASCULINO

II) ESTADO CIVIL:

a) ( ) $\operatorname{SOLTEIRO}(\mathrm{A})$

b) ( ) CASADO(A) / MORA COM UM(A) COMPANHEIRO(A)

c) ( ) SEPARADO(A) / DIVORCIADO(A) / DESQUITADO(A)

III) SUA IDADE ESTÁ ENTRE:
a) ( ) 15-19 ANOS
b) ( ) 20-24 ANOS
c) ( ) 25-29 ANOS
d) ( ) 30-34 ANOS
e) ( ) 35-39 ANOS
f) ( ) 40-44 ANOS
g) ( ) ACIMA DE 44 ANOS

\section{SOBRE O USO DO R.e.C.}

IV) VOCÊ CONHECE O SOFTWARE R.e.C?

a) ( ) SIM E JÁ O UTILIZEI EM SALA DE AULA.

b) ( ) SIM, MAS NUNCA O UTILIZEI EM SALA DE AULA

c) ( ) NÃO

V) SE VOCÊ RESPONDEU LETRA “a”, NA QUESTÃO 2.1, AINDA PRETENDE CONTINUAR A UTILIZAR O R.e.C., EM SALA DE AULA?
a) ( ) SIM

b) ( ) NÃO 
VI) SE VOCÊ RESPONDEU LETRA “b”, NA QUESTÃO 2.1, PENSA, EM ALGUM MOMENTO, UTILIZAR O R.e.C. EM SALA DE AULA?
a) ( ) SIM
b) ( ) NÃO

\section{SOBRE A SUA FORMAÇÃO PROFISSIONAL}

VII) AO LONGO DE SUA TRAJETÓRIA PROFISSIONAL, VOCÊ JÁ TEVE CONTATO COM SOFTWARES MATEMÁTICOS?

a) ( ) SIM

b) ( ) NÃO

VIII) SE VOCÊ RESPONDEU LETRA “a”, NA QUESTÃO 3.1, ONDE OCORREU?
a) ( ) NA ESCOLA
b) ( ) NO AMBIENTE DE TRABALHO
c) ( ) OUTROS

IX) SE VOCÊ RESPONDEU LETRA “a”, NA QUESTÃO 3.1, FOI TRABALHADO COMO UTILIZAR O SOFTWARE EM SALA DE AULA?
a) ( ) SIM
b) ( ) NÃO

X) VOCÊ TEM CONHECIMENTO DE POLÍTICAS PÚBLICAS NA SUA ESCOLA QUE INCENTIVAM O USO DE SOFTWARES EDUCACIONAIS PARA USO EM SALA DE AULA?
a) ( ) SIM
b) ( ) NÃO
OUTRA RESPOSTA:

XI) NA SUA ESCOLA VOCÊ SE SENTE DESAFIADO A PESQUISAR E PROCURAR OUTROS SOFTWARES EDUCACIONAIS?
a) ( ) SIM
b) ( ) NÃO
c) OUTRA RESPOSTA: 
PAIM, M. A. S. O problema do carpinteiro: estudando semelhança de triângulos por meio da fachada de uma casa

\section{APÊNDICE B - Pré-teste}

\section{PRÉ - TESTE}

Duração da aula: 1 aula de 50 minutos

Objeto matemático de estudo: Semelhança de triângulos

Objetivo: Determinar a medida do segmento de um lado de um triângulo.

Determine o valor do segmento AE na figura a seguir:

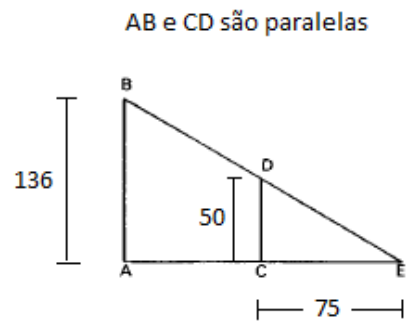

Figura 9 - Exercício para determinar o segmento AE Fonte: Adaptado de Iezzi (1993, p. 400) 


\section{APÊNDICE $\mathrm{C}-\mathrm{A}$ atividade}

\section{ATIVIDADE: O problema do carpinteiro}

Duração da aula: 2 aulas de 50 minutos

Objeto matemático de estudo: Semelhança de triângulos

Objetivo: Obter razões de semelhança entre triângulos.

Objetivos específicos:

- Utilizar ferramentas do software R.e.C. para a resolução de problemas;

- Determinar segmentos de reta correspondentes aos lados dos polígonos.

Um carpinteiro pretende ajustar diferentes tipos de vidros em iguais janelas (espaços vazios em branco) que se encontram na fachada de uma parede da sua casa. Para isso, precisa saber quais são os possíveis valores das dimensões dos seus lados:
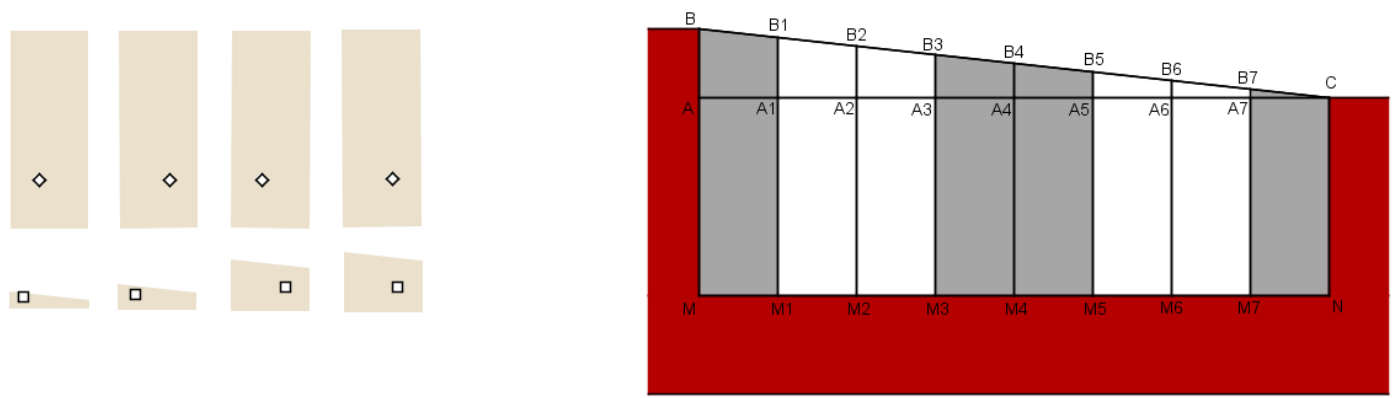

Figura 10 - O problema do carpinteiro Fonte: Elaborada pelo autor

Com o auxílio do software R.e.C., ajude o dono a alcançar o seu objetivo, seguindo os passos indicados e respondendo às questões propostas:

1) Acione a ferramenta ponto $\circ$ e deslize com o mouse em cima dos lados de cada janela da lateral da casa. Ao fazer isso, perceba que surgirá um ponto de cor laranja em cada lado. Clique, então, com o botão direito do mouse em cima de cada segmento. O que você percebeu ?

2) Cite exemplos de segmentos de reta com a mesma medida. 
3) Complete a tabela abaixo de acordo com alguns dos valores obtidos anteriormente, com uma casa decimal.

\begin{tabular}{|c|c|c|c|c|c|c|}
\hline Segmentos & AB & A1B1 & A2B2 & A3B3 & A4B4 & A5B5 \\
\hline Comprimentos & & & & & & \\
\hline
\end{tabular}

4) Com a ferramenta , escolha o segmento indicado e complete a seguinte tabela com uma casa decimal:

\begin{tabular}{|l|l|l|l|l|l|}
\hline BC & B1C & B2C & B3C & B4C & B5C \\
\hline & & & & & \\
\hline AC & A1C & A2C & A3C & A4C & A5C \\
\hline & & & & & \\
\hline
\end{tabular}

O que é comum à todos os segmentos?

5) $\mathrm{O}$ segmento $\mathrm{MN}$ se divide em 8 segmentos iguais? $\mathrm{O}$ mesmo vale para $\mathrm{AC}$ e $\mathrm{BC}$ ? Justifique.

6) Novamente com a ferramenta ponto $\circ$, clique, respectivamente, sobre os pontos B, C e A. Acione a ferramenta ângulo com amplitude fixa dô e observe o tamanho do ângulo. Repita o mesmo procedimento para B1, C, A1, para B2, C, A2, e assim sucessivamente. O que é possível afirmar?

7) Com uma calculadora, obtenha os valores das razões indicadas na tabela:

\begin{tabular}{|c|l|l|l|}
\hline triângulos & \multicolumn{3}{|c|}{ razões } \\
\hline $\mathrm{ABC}$ & $\mathrm{AB} / \mathrm{BC}=$ & $\mathrm{AB} / \mathrm{AC}=$ & $\mathrm{BC} / \mathrm{AC}=$ \\
\hline $\mathrm{A} 1 \mathrm{~B} 1 \mathrm{C}$ & $\mathrm{A} 1 \mathrm{~B} 1 / \mathrm{B} 1 \mathrm{C}=$ & $\mathrm{A} 1 \mathrm{~B} 1 / \mathrm{A} 1 \mathrm{C}=$ & $\mathrm{B} 1 \mathrm{C} 1 / \mathrm{A} 1 \mathrm{C}=$ \\
\hline $\mathrm{A} 2 \mathrm{~B} 2 \mathrm{C}$ & $\mathrm{A} 2 \mathrm{~B} 2 / \mathrm{B} 2 \mathrm{C}=$ & $\mathrm{A} 2 \mathrm{~B} 2 / \mathrm{A} 2 \mathrm{C}=$ & $\mathrm{B} 2 \mathrm{C} 2 / \mathrm{A} 2 \mathrm{C}=$ \\
\hline
\end{tabular}


PAIM, M. A. S. O problema do carpinteiro: estudando semelhança de triângulos por meio da fachada de

\begin{tabular}{|l|l|l|l|}
\hline $\mathrm{A} 3 \mathrm{~B} 3 \mathrm{C}$ & $\mathrm{A} 3 \mathrm{~B} 3 / \mathrm{B} 3 \mathrm{C}=$ & $\mathrm{A} 3 \mathrm{~B} 3 / \mathrm{A} 3 \mathrm{C}=$ & $\mathrm{B} 3 \mathrm{C} 3 / \mathrm{A} 3 \mathrm{C}=$ \\
\hline $\mathrm{A} 4 \mathrm{~B} 4 \mathrm{C}$ & $\mathrm{A} 4 \mathrm{~B} 4 / \mathrm{B} 4 \mathrm{C}=$ & $\mathrm{A} 4 \mathrm{~B} 4 / \mathrm{A} 4 \mathrm{C}=$ & $\mathrm{B} 4 \mathrm{C} 4 / \mathrm{A} 4 \mathrm{C}=$ \\
\hline $\mathrm{A} 5 \mathrm{~B} 5 \mathrm{C}$ & $\mathrm{A} 5 \mathrm{~B} 5 / \mathrm{B} 5 \mathrm{C}=$ & $\mathrm{A} 5 \mathrm{~B} 5 / \mathrm{A} 5 \mathrm{C}=$ & $\mathrm{B} 5 \mathrm{C} 5 / \mathrm{A} 5 \mathrm{C}=$ \\
\hline
\end{tabular}

De acordo com os dados da tabela, qual a sua conclusão sobre os triângulos? E sobre as razões?

8) Com os botões apropriados : $\square$ ou $\diamond$, clique e arraste os vidros para as suas respectivas janelas 